\title{
HEALTH HAZARDS OF THE PHARMACEUTICAL INDUSTRY
}

\author{
BY \\ R. M. WATROUS \\ From the Abbott Laboratories, North Chicago, Illinois, U.S.A.
}

(RECEIVED FOR PUblCATION, NOVEMBeR 25, 1946)

In recent years the pharmaceutical industry has come to occupy a unique position in regard to problems of industrial hygiene and toxicology ; practically no other single commercial enterprise presents such a wide variety of potentially toxic exposures or such a rapidly-changing advent of new chemical substances. This dynamic situation, which renders the work of the plant physician so instructive in this industry, has been created by the increasing application of organic chemical synthesis as a method for producing therapeutic substances. Around the core of the old industry, which was chiefly concerned with extracting active ingredients from medicinal herbs, there has been built up in the past twenty years a new and highly-complicated fine chemical manufacturing enterprise, concerned with intricate organic syntheses, and dependent for its life on being able to follow the most advanced techniques developed in the research laboratory. Such manufacture, which seldom settles down to the sober mass-production of the heavy chemical industry, is essentially a batch-process and half-experimental business, full of unexpected occurrences, strange chemical substances, and unusual toxicological problems. It is rarely able to make use of manufacturing equipment specifically designed for one synthesis; that is a privilege reserved for heavy chemicals. As a consequence, more versatile equipment must be used-equipment which must always fall short of the ideal in either safety or efficiency where any specific synthesis is concerned.

This. concretion of the new industry around the old makes a convenient division in the consideration of toxicological hazards: the problem of handling crude vegetable drugs and their extracts may be considered separately from the more complicated problems of synthetic chemistry in the factory. Under a third heading certain miscellaneous hazards to health will be considered.

\section{Hazards from Handling Crude Drugs and Their Extracts}

The hazards of extracting active principles from crude drugs are unequally divided between the three classes of materials involved; the herbs themselves, the solvents, and the finished active material. Of the three, the crude materials offer the least striking dangers to health. Two exceptions which have been prominent in my own experience are ipecacuanha and podophyllum.

Ipecacuanha.-The powdered form of the former may act as a mild primary irritant on the skin and mucous membranes, but is especially dangerous to those who have developed an allergic sensitivity to it; asthma specifically caused by this herb has been described (Salter, 1860; Peshkin, 1920). I have seen one nearly fatal asthmatic seizure provoked by inhalation of very small amounts of dust from the powdered drug.

Podophyllum.-Podophyllum root is still handled in large amounts as a source of the benzene extract, podophyllin, a time-honoured laxative. The irritant resin which constitutes the active principle is present in sufficient amounts in the dry powdered root to constitute a hazard. Workers who feed the root into the grinder must be protected by goggles and dust respirators to prevent irritation of the mucous membranes. In exceptional cases, even the skin may be irritated by the dust. Failure to use protective equipment results in a characteristic, very painful conjunctivitis and keratitis, which is delayed in its onset for four to six hours after exposure and lasts several days. In its mild form this irritation subsides spontaneously under treatment with antiseptic and local anaesthetic ointments; a more severe form, 
caused by massive exposure to the concentrated podophyllin, may produce diffuse infiltration of the cornea, or even ulceration.

Penicillin.-The extraction of penicillin from mould cultures, though more modern in date, nevertheless properly should be classified with older processes of pharmaceutical manufacturing. It has, in my experience, presented one exposure which, though not toxic, had a sufficient nuisance value to require medical investigation. The first penicillin was grown in shallow trays. At one time, many of the cultures were being contaminated by Aspergillus niger, which matured and sporulated abundantly. The contaminated cultures were worthless, but had to be dumped in order to sterilize and re-use the pans. The dumping released dense clouds of black spores into the air; the workmen inhaling the dust found their nasal mucus and their sputum stained black for many hours thereafter, and were thus thrown into a mild panic. It was difficult to reassure these men on the grounds that the mould was probably non-pathogenic, especially since nothing in the literature could be found concerning exposures of similar magnitude. However, the passage of time and the continued good health of the workmen disposed of all our fears, and the advent of deep-tank culture methods rendered this particular nuisance obsolete.

\section{Solvents}

The solvents used to extract active principles from crude drugs are numerous and varied. Methyl alcohol is not commonly used. Ethyl alcohol offers medical hazards only to the extent to which it is denatured with more toxic materials.

12- $A$. The most available form of anhydrous ethyl alcohol in the United States is formula 12-A, which contains 5 per cent. of benzene. The hazards of this material arise from the tendency of chemical workers to regard it as alcohol pure and simple, and to handle it with corresponding carelessness. To the industrial physician it presents another difficulty: measurements of its concentration in the air are complicated by the presence of the alcohol. Instruments based on the combustible vapour principle give readings which can be interpreted in terms neither of pure alcohol nor pure benzene, and the colour development in the butanone test is affected by the presence of the alcohol. However, I am able to report that a group of two or three workers who were employed for some five years in almost daily contact with concentrations of 12-A alcohol vapour sufficient to cause stinging of the eyes and an overpowering odour failed to show any change in blood counts, or other signs of ill-health. Butanone tests made during the heaviest exposure showed concentrations of benzene ranging from 20 to 100 parts per million, as nearly as could be estimated with the anomalous colour-development.

Benzene.-Benzene itself is, unfortunately, such an excellent solvent for many active principles, as well as for numerous important synthetic chemicals, that it constitutes a major problem in the pharmaceutical industry. This is not the place to describe or discuss the well-known facts about acute and chronic benzene poisoning, except to affirm from my own experience that these well-established facts are still completely unknown or disregarded in more backward works, and that they cry out for more popular diffusion among workmen and foremen, even in the most progressive organizations. Accordingly, this review will present only those observations on the control of chronic benzene poisoning which have proved useful or pertinent in my own experience.

This experience covers eight years' observation of a group of some 150 workmen in a chemical manufacturing department, in which benzene is handled in scores of processes, in amounts ranging from a few litres to a thousand gallons, and under conditions ranging from the very best closed methods to the most primitive and dangerous ones. The exposures are essentially intermittent; grab samples of air have shown concentrations as high as 3,000 parts per million, and as low as 0 parts per million. Shifting men from one process to another has been a characteristic feature of this department; consequently, though some work periods involve no exposure to benzene for months, each man must be observed for signs of poisoning at all times, since all are exposed at one time or another-and to unpredictable degrees.

The mainstay of preventive medicine in this situation continues to be periodic determination of the blood count of each man every three months. If suspicious or abnormal values are observed, the interval of examination is shortened, and investigation of the clinical and occupational details of the individual case is undertaken. The details of this system are described in full elsewhere (Watrous, 1943a). Suffice it to say here that arbitrary limits have been set up to define suspicious and abnormal values of the blood counts. In fixing these limits, the industrial physician should bear in mind the normal variation which exists in all biometric data; this variation can be conveniently visualized by constructing frequency-distribution curves for the various quantities measured. In my opinion, no other method approaches this one in completeness and ease of comprehension, and it should be universally used to describe blood-count statistics for any group. Fig. 1 is a series of frequency-distribution curves for my group of workers, covering the period May, 1945, to August, 1946, and representing 880 complete blood counts on 200 individuals. 


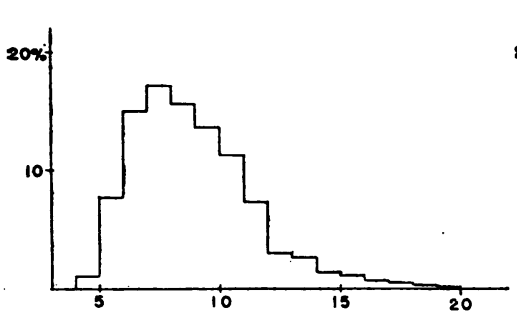

W.B.C.- THOUSANDS PER CU. MM.
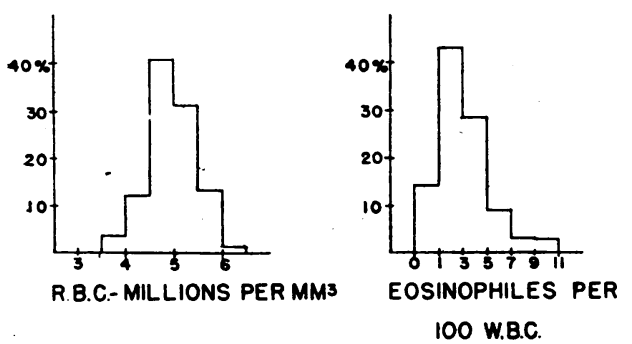

Fig. 1
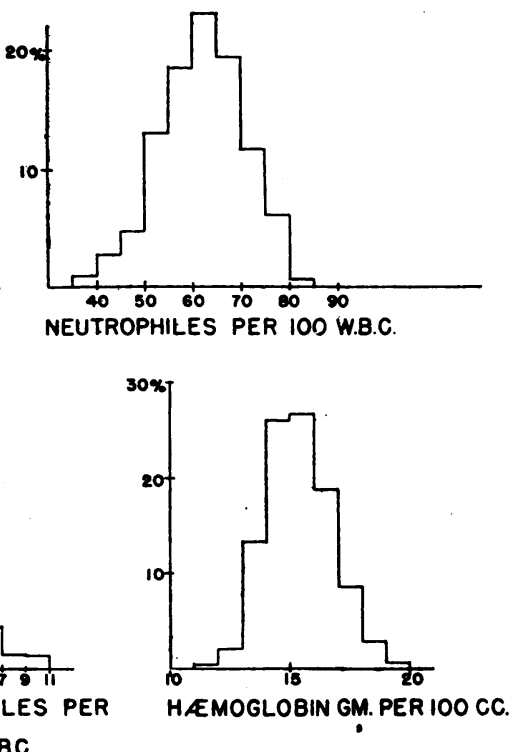

NEUTROPHILES PER 100 W.B.C.

except to indicate that a given individual has absorbed benzene during a given period. Because of the enormous variation in sensitivity of individuals to benzene exposure, proof of absorption is no indication of damage, just as a normal sulphate ratio is no guarantee of absence of damage. The condition of the bone marrow is of the greatest importance, and this is most accurately reflected in the blood count. However, mere passive waiting for signs of bone-marrow damage to appear is no adequate programme for the prevention of benzene poisoning ; it is, rather, an indispensable last line of defence. If no other division of the industry is charged with the task of

Though other investigators (Greenburg and others, 1939) have found that changes in the size of the erythrocytes and very mild anaemia are the earliest haematological signs of benzene poisoning, I have not found these indices reliable. The most constant early sign of poisoning, in my experience, has been a progressive decline in the total white count to values below 5,000 per c.mm. I have not found this to be accompanied by any consistent alteration in the ratio of lymphocytes to granulocytes, or any definite trend in the erythrocyte count or haemoglobin content of the blood. Leukopenia persisting below 5,000 cells per c.mm. has been my criterion for complete removal of the worker from the chemical manufacturing department; such measures have been required seven times in eight years, and in each case the blood returned to and remained normal. The justification for this method of detecting benzene poisoning, and for the arbitrary values adopted as suspicious and abnormal, rests, therefore, on this limited experience. All that can be said for the method is that it has apparently prevented the occurrence of any case of irreversible bone-marrow damage over an eight-year period.

In my limited experience, determination of urinary sulphate ratios has not been useful. The fact that exposure to benzene is present may be determined by the physician's nose as he walks through the works, and may be proved quantitatively at any given time and place by air sampling. Urinary sulphate ratios add nothing to this knowledge, inspection of the work-place, air sampling, and the design of closed or ventilated equipment, the industrial physician. must assume these duties. In any case, he must be fully informed of all activities relating to industrial hygiene and preventive engineering which take place in the factory, and should play an active part in furthering such work.

Chloroform and ethylene dichloride.-Chloroform and ethylene dichloride (1,2-dichloroethane) are occasionally used to extract alkaloids and other fat-soluble principles. Both solvents are liver poisons, and men working with them under ordinary conditions may develop certain vague symptoms which are referable to the gastrointestinal tract and which suggest very slight liver damage. These consist of anorexia, a heavy feeling in the epigastrium, and fatigue. At this time the urine may give a positive test for urobilinogen, and in my experience this is an indication for transferring the workman temporarily to other employment. Amyl acetate and ethyl ether are other solvents occasionally encountered in vegetable extractions. Neither has proved especially toxic in my experience; the former because its strong odour has a deterrent effect on those who are in danger of overexposure, the latter because of its very low inherent toxicity.

\section{Alkaloids}

The purified extracts of vegetable drugs include all the alkaloids which are used in therapeutics: atropine, hyoscine, scopolamine, emetine, aconitine -all powerful poisons which may cause illness if handled by careless or untrained workers. A common sight in the medical dispensary of a pharma- 
ceutical industry, and a baffling one for the novice, is the single widely-dilated pupil caused by the entrance of minute particles of one of the belladonna alkaloids into the worker's eye in the form of dust. This may be seen even in persons who merely walk through the manufacturing department. One application of eserine $\frac{1}{4}$ per cent. ointment to the affected eye, after thorough irrigation with normal salt solution, will usually correct the pupillary dilatation within a few hours.

It is quite possible for a workman, if improperly instructed and protected, to develop acute poisoning by inhaling the dust of pure alkaloids of the belladonna group. One such case which I observed was that of a chemical worker 31 years old who had been grinding pure atropine sulphate through a fine-mesh sieve. His foreman had failed to impress upon him the necessity of wearing a dust respirator. He presented himself in a mildly inebriated state, complaining of blurred vision and dryness of the mouth. The pupils were widely dilated, the skin was flushed, and the pulse was 132. The patient was persuaded, with some difficulty, to rest in bed, where he tossed about, talking incessantly in a disoriented fashion. It was decided to administer eserine or pilocarpine in fractional doses intravenously, and here we encountered a difficulty which all physicians in charge of pharmaceutical works would be well advised to keep in mind: there was no preparation of eserine or pilocarpine at hand in a form suitable for parenteral injection.* The emergency was met by asking the pharmacologist's laboratory to weigh out and dissolve eserine in the same way as they would do for animal experiments. Repeated doses of $1 \mathrm{mg}$. were injected intravenously, each injection being followed by a period of observation, in which the condition of the pulse and the pupils was the most informative index of the patient's condition. After four injections the pulse had slowed to 102 and the patient was out of danger. There were no untoward after-effects, and the man was back at work the next day.

Aconitine hydrobromide may also give rise to acute poisoning if handled in the form of the pure powder. In one mild case which I observed, the symptoms were identical with those produced by a strong cigar in a person not used to smoking: there were pallor, weakness, nausea and vomiting, and cold sweat. The illness subsided in about thirty minutes; it was caused by the inhalation of dust while weighing a small amount of aconitine hydrobromide on an ordinary laboratory balance without using protective equipment.

In my experience emetine hydrochloride has never produced industrial systemic poisoning, but it is a local irritant; not infrequently, workers filling ampoules with the 6.5 per cent. solution may

* Prostigmine, or prostigmine methyl sulphate, are suitable for this purpose, and should be kept available wherever belladonna alkaloids are being handled. splash a drop in one of their eyes. A painful chemical conjunctivitis results, which requires about 48 hours to subside.

One unusual toxic exposure which came to my notice was the result of weighing some $500 \mathrm{~g}$. of pure desiccated scarlet fever toxin, prepared by the method of George and Gladys Dick. The light, fluffy powder was readily dispersed in the form of dust, but no one had thought to warn the pharmacist about protection against this hazard. He presented himself the next day with a sore throat, a typical strawberry tongue, and an erythematous rash. There was no fever; repeated tests revealed no albumin in the urine. The symptoms had subsided the next day. The patient had never had scarlet fever.

\section{The Hazards of Organic Synthesis}

Organic chemical synthesis presents industrial hazards of three main types. Firstly, the active agents used to attack and modify the structure of organic compounds are, by their very nature, exceptionally able to attack and modify the organic compounds of the human body, thus producing highly poisonous effects. Secondly, the intermediate compounds in most organic syntheses are often characterized by the readiness with which they enter into chemical combination with other organic matter; they are active. This often confers toxic properties of great variety upon them. Thirdly, the final products, though they are medicines designed to be introduced into the human body, may nevertheless produce severe poisoning under conditions of industrial exposure.

\section{Chlorinating Agents}

Of the active agents widely used in organic synthesis, the chlorinating agents form a group which very commonly produces illness when improperly used. The common members of this group include chlorine, phosphorus oxychloride $\left(\mathrm{POCl}_{3}\right)$, carbonyl chloride or phosgene $\left(\mathrm{COCl}_{2}\right)$, thionyl chloride $\left(\mathrm{SOCl}_{2}\right)$, and phosphorus pentachloride $\left(\mathrm{PCl}_{5}\right)$. Chlorine and phosgene are gases, phosphorus oxychloride and thionyl chloride are liquids which vaporize readily at normal temperatures, and phosphorus pentachloride is a solid, of which I have had no personal experience. The first four are lung irritants and, therefore, may produce pulmonary oedema if inhaled in sufficient amounts. Their warning properties vary. Chlorine gives good warning of its presence; thionyl chloride and phosphorus oxychloride cause considerable irritation of the mucous membranes, but may be said to have only fair warning properties; phosgene gives 
very poor warning. The most excellent engineering is required to prevent accidents when these chemicals are used in a manufacturing process, since, in addition to being poisons, they are highly corrosive to common metals; ordinary ventilating equipment, condensers, pipes, and tanks are apt to be destroyed within a few weeks. The leakage which may then result is extremely dangerous. Ordinary canistertype masks are not safe in such circumstances; repair workers should be equipped with air-supply masks or closed oxygen systems.

\section{Sulphonating Agents}

Of the sulphonating agents, chlorsulphonic acid $\left(\mathrm{HOSO}_{2} \mathrm{Cl}\right)$ is extensively used in the manufacture of $p$-acetylaminobenzene sulphonyl chloride, a necessary intermediate for most sulphonamides. The fumes of the acid itself are highly irritating, and in many sulphonation reactions $\mathrm{HCl}$ gas and $\mathrm{SO}_{2}$ are given off. It is often not economically feasible to trap these irritant by-products in a small synthesis, and they are often vented into the outside air. This is a bad practice, which will cause a large amount of bronchitis and conjunctivitis under adverse wind and weather conditions. Scrubbing towers of simple and cheap design, or very high stacks, are usually required to eliminate the nuisance. Other common sulphonating agents, concentrated $\mathrm{H}_{2} \mathrm{SO}_{4}$ and fuming sulphuric acid (oleum), are too familiar to require much discussion. However, it is well to remember that the heat of reaction of oleum and water is so great that there is some question as to the proper method of washing this acid off the skin, if it should be spilled. We recommend liberal dousing with anhydrous ethyl alcohol for the first wash, to be followed immediately by streams of water.

\section{Methylating Agents}

A common methylating agent used in the pharmaceutical industry is dimethyl sulphate $\left(\left(\mathrm{CH}_{3}\right)_{2} \mathrm{SO}_{4}\right)$. It is a highly corrosive, volatile liquid, possessing vesicant and lung-irritant properties. It must be handled with the same care as the liquid chlorinating agents described in a previous section. Its warning properties are only fair.

The laboratory chemist is sometimes tempted to use two methylating agents of extreme toxicity because they make possible certain very elegant syntheses. These are nitrosomethyl urethane and

diazomethane.<smiles>CCCCCN(C)C(=O)ON=O</smiles>

The latter may be obtained by treating the former with alcoholic $\mathrm{KOH}$, and is an extremely poisonous, yellow, odourless gas, described in the German literature of 1895 , but having received scant attention since then. Von Pechmann (1895) stated that nitrosomethyl urethane caused ' red, itching areas and blisters to appear on the skin. Inhalation of its sweetish vapour causes severe bronchial catarrh, as well as a painful irritation and disturbance of accommodation of the eyes. Since these symptoms are not unlike those produced by diazomethane. it seems probable that the toxicity of nitrosomethyl urethane is due to its conversion to diazomethane in the body.'

The difficulty which these compounds would present if used in large-scale production may be imagined by considering the effects which I observed in two laboratory chemists who made up a few ounces to use in an experiment. In both cases the chemical was spilled on the operator's hands. There appeared to be a latent period of five to ten days between the exposure and the appearance of the dermatitis. The first symptom was a painless erythema which, after two to four days, developed into a severe, itching, uniform erythema, with marked swelling of the skin. At this time the skin assumed a peculiar dusky, cyanotic appearance, and itching was pronounced, No blisters appeared at any time. In the severe case, the phase of acute swelling lasted about five days and then subsided, being followed by an extensive desquamation of the affected skin.

These two cases, fortunately, represent my only experience with this compound, since it has never been thought feasible to introduce it into large-scale manufacture. I was informed, however, by the director of synthetic chemistry of another large American chemical works, that whenever they manufactured nitrosomethyl urethane, reactions occurred in the workers despite all possible precautions. They were finally obliged to discontinue manufacture of the compound for this reason.

Experiments performed by R. K. Richards (personal communication) demonstrated that nitrosomethyl urethane painted on the shaved skin of rats at twentyfour-hour intervals will kill the animals after two applications. Purplish discoloration of the skin appeared in one of three animals. Death was evidently due to haemorrhagic oedema of the lungs, probably caused by inhalation of vapour from the skin. Nitrosomethyl urea $\left(\mathrm{H}_{3} \mathrm{CN}-\mathrm{C}-\mathrm{NH}_{2}\right)$, another compound<smiles>N#CN=O</smiles>

from which diazomethane may be generated, showed no adverse effects when painted on the skin of rats in a 10 per cent. alcoholic solution for four successive days. However, one case of severe dermatitis from handling this compound has been reported to me (Hager, personal communication). 


\section{Alkyl Halides}

The alkyl halides are extensively used in synthetic chemistry for the purpose of adding methyl and other alkyl groups to compounds, by the general reaction: $R-N a+R^{\prime} X \rightarrow R-R^{\prime}+N a X$. They find extensive use, particularly in the synthesis of barbituric acid derivatives, in which a large variety of alkyl groups are linked to the middle carbon of the malonic acid or the cyanoacetic molecules:

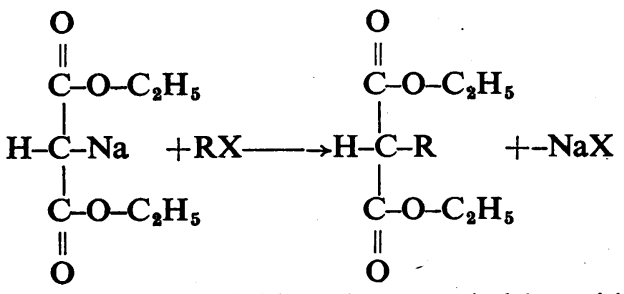

Common members of this series are ethyl bromide, $n$-butyl bromide, isopropyl bromide, 1-methylbutyl bromide, and many others, limited in variety only by the number of alcohols which may be usefully substituted in the barbiturate molecule. Methyl bromide and benzyl chloride find application in other syntheses. The literature dealing. with the toxic properties of these compounds as a group is limited largely to reports of experiments on animals (Flury and Zernik, 1931), but a few of the more noxious members of the series have given sufficient trouble to cause extensive comment for themselves individually.

Methyl bromide.-Leading the list is methyl bromide, unquestionably the most toxic, which may produce disturbing symptoms in concentrations below 35 parts per million (Watrous, 1942). I have had no experience with this compound in its capacity as a methylating agent, but at one time during the war a number of pharmaceutical manufacturers in the United States were requested to fill the liquid into glass ampoules for military use as a delousing agent. Widespread difficulty was encountered at that time, not only from inhalation of the vapour, but especially from the vesicant action of the liquid, which had previously been reported in the literature but was not widely known at that time. This vesicant property is also present in benzyl chloride, but is absent in ethyl bromide, isopropyl bromide, and 1-methyl-butyl bromide. I have observed a small group of workers exposed intermittently to ethyl bromide vapour in concentrations up to 1,500 parts per million, to benzylchloride up to 500 parts per million, and to 1-methyl-butyl bromide up to 920 parts per million. No systemic symptoms were complained of by these men over a period of years, though the vapour of benzyl chloride produced some conjunctivitis and irritation of the upper respiratory tract in a few instances. However, no one who has witnessed the insidious and deadly action of methyl bromide, with its delayed effect upon the central nervous system, can regard any of the lower alkyl bromides with anything but distrust, and I believe that closer observation of men chronically exposed to these compounds may reveal mild degrees of damage. In any event, most members' of the alkyl halide series are powerful anaesthetic agents, and must be handled with caution for that, if for no other reason.

Sodium and potassium cyanide.-Sodium and potassium cyanide are frequently used in organic synthesis to form nitriles with organic halides:

$$
\mathrm{NaCN}+\mathrm{RX} \longrightarrow \mathrm{RCN}+\mathrm{NaX}
$$

It is a fact, not peculiar to the pharmaceutical industry, that the cyanides themselves, despite their great toxicity, seldom are the cause of serious poisoning under ordinary conditions of use. Such poisoning as does occur is usually the result of an accident in which acid is allowed to come in contact with unreacted cyanide. In such an event, some of the largest handlers of cyanide in the United States favour the antidotal measures described by Chen and others (1944), in which $10 \mathrm{c.cm}$. of 3 per cent. sodium nitrite are injected intravenously, followed by $50 \mathrm{c} . \mathrm{cm}$. of 25 per cent. sodium thiosulphate. Boxes containing all the necessary materials for such treatment are sometimes prepared and kept ready for emergencies in the work-rooms where cyanide is handled. Many of the nitriles have toxic properties resembling cyanide itself, and in general they must be handled, if volatile, with extreme care. In one case of exposure to benzyl cyanide (benzonitrile) which I observed, the symptoms consisted of vertigo, faintness, ataxia, pallor, and weak pulse, followed by headache and vomiting the next day. The general properties of this compound are described by Heffter (1923).

\section{Acetylating Agents}

Acetylating agents used in organic synthesis are numerous. Glacial acetic acid, acetic anhydride, monochloracetic acid, and dichloracetic acid are a few. These compounds, in varying degrees, have the peculiar property of producing extensive bullous desquamation of the skin, especially on the palms of the hands, with little or no warning. It is not uncommon to see separation of the entire upper layer of the skin on both palms in workmen who spill these substances on their hands. In most cases the victim is unaware of anything except a mild stinging or itching sensation and, therefore, does not trouble to wash off the chemical or take any other action to 
halt its destructive effect. When such accidents are reported promptly, soaking the affected parts for as long as four hours in sodium bicarbonate solution will generally prevent any damage; these very protracted soaks are essential to permit the reagent to diffuse out of the skin instead of into it. The acetylating agents are, of course, also lung irritants, but their warning properties are such that pulmonary complications are seldom seen.

\section{Condensing Agents}

What might be called the condensing agents form a miscellaneous group of considerable chemical activity. Sodium methylate and sodium ethylate are widely used in the chemical synthesis of drugs. They are usually made up just before use by adding sodium to the appropriate alcohol in a closed tank or still; the next step in the reaction is then usually carried out without delay in the same container. In this way, the workmen are never directly exposed to the compound except by some unusual accident. The chief danger from the compounds themselves is their corrosive effect upon tissues. The hazard in making them arises from the presence of sodium metal and hydrogen, with the possibility of explosion and fire if improper methods are used. Concentrated sulphuric acid, phosphorus oxychloride, phosphorus pentachloride, and phosphorus pentoxide are often used as condensing agents because of their extreme avidity for water. The same property endows them with corrosive properties in respect to human tissue, most of which have been previously discussed.

\section{Nitrating Agents}

Nitrating agents find occasional use in the pharmaceutical industry, though in general these reactions are more common in the heavy chemical industry which supplies the basic chemicals from which fine chemicals are made. The dangers of corrosive action, explosion, and inhalation of $\mathrm{NO}_{2}$-the inevitable accompaniments of any nitration-are so familiar as to need no further comment.

\section{Organic Reductions}

Organic reductions are common in the synthesis of medicinal chemicals, and are most usually brought about by the addition of finely-divided iron or zinc, with an acid, to the organic substance. The evolution of hydrogen always creates the hazard of explosions, and the storage, handling, and recovery of finely-powdered zinc carries with it certain dangers which are not commonly recognized. Dry powdered zinc, if dispersed as a dust in air, is highly explosive within certain limits; wet powdered zinc may undergo oxidation and is subject to spontaneous combustion (Brown, 1941).

\section{Toxic Intermediate Compounds}

To describe all the toxic intermediate compounds which might be encountered in the organic syntheses peculiar to the pharmaceutical industry would require much space, and a great deal more unpublished knowledge than any one individual is likely to acquire at the present time. I shall, therefore, limit the following discussion to compounds of which I have personal knowledge or experience, and to those which present especially difficult or littleknown properties.

Dichlorethyl acetate.-In one method of synthesizing sulphathiazole, the intermediate compound aminothiazole is made by adding chlorine to vinyl acetate and by condensing the resultant dichloroethyl acetate with thiourea. Dichloroethyl acetate is an unstable liquid with a sharp odour, possessing vesicant and lung-irritant properties of somewhat lesser degree than mustard gas. It produces conjunctivitis and tracheitis in low concentrations in the vapour phase. Liquid leaking from the equipment may produce blisters; small amounts of liquid entering the eye will cause corneal ulceration.

Aminothiazole.-Aminothiazole itself is a crystalline solid with a marked tendency to. sublime at moderately elevated temperatures. Exposure to its vapour and dust causes dermatitis, some gastrointestinal symptoms, and a distinctive dark-brown pigmentation of the urine, not due to bile pigments or haemoglobin. Deichmann and his co-workers have investigated the toxicity of aminothiazole. They found that aminothiazole repeatedly inhaled at levels of $0.2 \mathrm{mg}$. per litre had a toxic effect on the lungs, liver, kidneys, and heart of guinea-pigs. The tissues of rabbits and rats exposed similarly demonstrated insignificant gross lesions; microscopic examination disclosed mild toxic degenerative changes in the liver and kidney. Two guinea-pigs which died after four and forty-one seven-hour exposures to an atmosphere containing $0.025 \mathrm{mg}$. per litre were found to have oedema, congestion, and emphysema of the lungs, and fatty degeneration of the liver. Three guinea-pigs which survived fortytwo such periods of exposure showed only slight hepato-cellular degeneration.

In workers exposed to the chemical in amounts varying from 0.1 to $3.0 \mathrm{mg}$. per cu. ft. $(0.0036$ to $0.11 \mathrm{mg}$. per litre), Watrous (1943b) found no evidence of liver damage, but occasionally there were anorexia, nausea, and vomiting. In three instances, a distinctive syndrome of urticaria followed by arthralgia appeared and ran a course lasting up to five weeks. The disorder closely resembled serum sickness. Further cases were prevented by 
adopting improved method of handling the chemical. The handling of 2-aminopyrimidine, a material of somewhat similar properties and an intermediate in the synthesis of sulphadiazine, produced no such symptoms.

Astwood and his co-workers (1945), in their investigation of compounds possessing anti-thyroid effect, found aminothiazole to be about oneseventh as active on the thyroid gland as thiouracil. This property was discovered independently in France by Perrault and his collaborators (1944-5), who observed symptoms of hypothyroidism in a group of aminothiazole workers. The chemical is now reported (J. Amer. med. Ass., September 28, $1946)$ to be in use in France as a medication for thyrotoxicosis, and some of the side-effects resemble the symptoms I observed in chemical workers. Three of my patients complained of excessive gain in weight, puffiness of the face and hands, and lassitude, but objective evidence of thyroid hypofunction was not definite enough to attract my attention at the time.

p-acetylaminobenzene sulphonyl chloride.--p-acetylaminobenzene sulphonyl chloride is a necessary intermediate in the synthesis of almost all the sulphonamides. It is prepared by sulphonating acetanilid with chlorsulphonic acid. The resulting crude chemical is a light-brown powder, somewhat unstable in the presence of moisture, and having a strong smell of acetic acid. The crude material is purified, and must then be thoroughly dried. The chemical is a moderately powerful primary irritant to the skin and mucous membranes, and may produce conjunctivitis, rhinitis, bronchitis, and dermatitis. In one unusual case, it produced bronchial asthma with a specific wheal formation when the diluted material was applied to a scratch on the skin. This is a very rare phenomenon in connexion with simple chemical compounds, as it generally indicates the presence of specific reagins in the blood.

The patient was a man 33 years old, employed for ten: years in chemical synthesis. A few years previously, he had been exposed to the vapour of thionyl chloride in sufficient amounts to produce pulmonary oedema requiring treatment with oxygen. While recovering from this accident, he was exposed to the dust of $p$ acetylaminobenzene sulphonyl chloride, and gradually noticed an increasing susceptibility to this irritant, which finally provoked typical asthmatic attacks. He was shifted to a part of the factory at least 300 yards from the location where this chemical was prepared and dried, but whenever the wind blew in his direction from his former place of work, he would become dyspnoeic. On many occasions he was able to tell within ten minutes after the mechanical drier in the remote part of the factory had been placed in operation. A. 1:100 solution of $p$-acetylaminobenzene sutphonyl chloride in acetone was mixed with equal parts of water and applied to a scratch on the patient's forearm. Within a few minutes, erythema, itching, and wheal formation of the extent usually spoken of as four plus appeared at the site of the scratch.

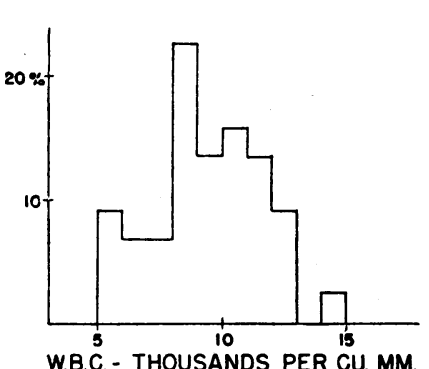

W.B.C - THOUSANDS PER CU. MM.

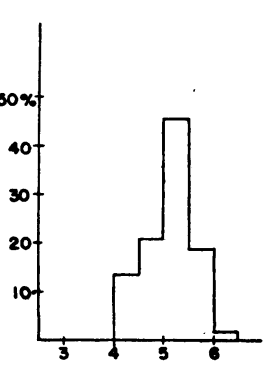

R.B.C.- MILLIONS PER MM3

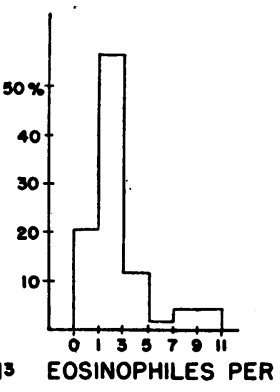

100 W.B.C.
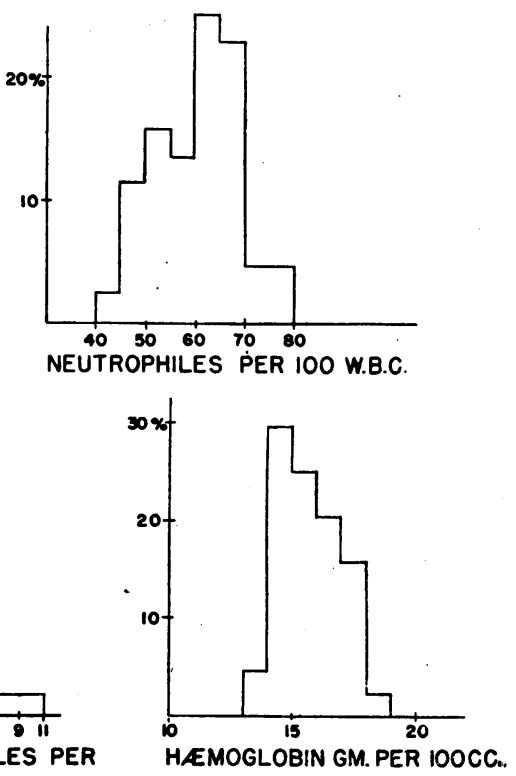

Fig. 2 
A control scratch not treated with the chemical showed no reaction. The same solution was applied to the skin of four normal subjects after scratching; no erythema or wheal formation occurred. A 1: 10,000 dilution of the chemical produced no reaction on the patient's skin or on the skin of controls.

A small series of cases exhibiting specific atophy to simple chemical compounds, with more elaborate proof of reagin formation, will be referred to in another section.

p-acetylaminobenzene sulphonyl chloride.-p-acetylaminobenzene sulphonyl chloride was claimed to be the cause of aplastic anaemia in a lawsuit brought in one of the eastern states of the U.S.A., and therefore data on the blood counts of workers handling this chemical are pertinent. Fig. 2 represents the frequency-distribution of figures from 43 blood counts made on 6 workmen who handled this chemical steadily from May, 1943, to May, 1944 . They were exposed to the crude material, the acetone solution, and the dust from the refined product. The concentration in the air was always less than 0.066 mg. per cu. $\mathrm{ft}^{*}{ }^{*}$ By comparing these curves with those in fig. 1 , it will be seen that no significant trend indicating depression of a haematopoietic activity can be detected. In the case mentioned, I was informed that the plaintiff had worked many years in another factory where there was exposure to benzene, and had only recently come in contact with $p$-acetylaminobenzene sulphonyl chloride in working for the company against which he filed suit.

Pentothal sodium.-Thiourea has already been mentioned as an intermediate in the synthesis of aminothiazole; it is also used in the manufacture of pentothal sodium. Thiourea is known to be a depressant of thyroxin formation. However, the dose required to produce this effect clinically is of the order of one or more grammes per day, and it is difficult to conceive of industrial exposure which would permit the absorption of this amount; in my experience, no toxic effect of any kind has occurred in men handling this compound. Organic compounds of sulphur in general tend to contain impurities of variable composition, usually foul-smelling, and of uncertain toxicity. Most of these compounds spontaneously liberate $\mathrm{H}_{2} \mathrm{~S}$, and it is difficult to ascertain, in any case, whether the symptoms of intoxication are predominantly caused by this or

* This concentration was present at the exhaust outlet of a rotary drier, and was too irritating to be tolerated in the air of the workroom. It was determined by collecting the chemical in an impinge containing strong aqua ammonia, thus forming acetyl sulphanilamide, which was measured, after neutralization and hydrolysis, by Marshall' a the dust appeared to consist of sulphanilic acid, and thus gave a test by Marshall's method withou hydrolysis. by other sulphur compounds. In the purification of crude Pentothal Sodium, $\mathrm{H}_{2} \mathrm{~S}$, mercaptans, and other disagreeable smelling compounds are present, and may produce headache, nausea, vomiting, faintness, and vertigo if not removed by proper ventilation.

Carbon monoxide.-Carbon monoxide is an expected by-product of certain organic syntheses, as in the manufacture of phenylethylmalonic ester. This ester is formed by the condensation of phenyl ethyl acetate with diethyl oxalate; when the condensate is distilled, $\mathrm{CO}$ is liberated. Since the gas is quite pure and odourless, it gives no warning of its presence. Points which must be carefully watched in the design of equipment for such distillations are: discharging condensed steam into sewers, thereby carrying trapped gas to remote points; leaks in the exhaust line of the vacuum pump; and the provision of water seals on all floor drains communicating with the chemical sewer. Carbon monoxide is also produced when methyl or ethyl formate are condensed with ethyl acetate in the preliminary stages of synthesizing 2-aminopyrimidine. This toxic gas is, of course, also to be reckoned with in hundreds of locations in the pharmaceutical factory in which illuminating gas is used for fuel. Special problems arise where many gas flames are used for sealing glass ampoules in rooms which must be kept free of flying dust, or in which the moisture-content of the air must be kept low; in such circumstances, conventional exhaust ventilation may be difficult or impossible to use.

Arsenic.-All the intermediates of arsphenamine and its derivatives are potentially toxic because they contain arsenic. All these compounds are solids, and must be handled in the form of dry powders, with the production of dust. I have been unable to find any study of the toxicity of these materials under the usual conditions of manufacture, but observations made by Watrous and McCaughey (1945) indicated that intermittent exposure to the dust of arsenical intermediates at levels ranging from 0.001 to $0.015 \mathrm{mg}$. per $\mathrm{cu}$. $\mathrm{ft}$. did not give rise to any symptoms of chronic arsenic poisoning over long periods of time, though the amount of arsenic excreted in the urine was higher than normal. Enormous amounts of arsenic were found in the hair of some of these workmen who had no symptoms of poisoning; this was considered to consist of settled dust, firmly attached to the hair, but might prove very misleading in any medico-legal cases because of the importance attached to arsenic in the hair by the conventional teachings of toxicology and forensic medicine. 
Aniline.-Aniline is an intermediate in the synthesis of arsanilic acid; dimethylaniline is used as a solvent in the synthesis of aminopyridine. The toxicity of the former can hardly be over-exaggerated, since the accepted maximum allowable concentration of the vapour is scarcely more than that for such dangerous poisons as $\mathrm{HCN}, \mathrm{H}_{2} \mathrm{~S}$, and $\mathrm{CS}_{2}$. The danger to untrained workers is more likely to consist of disregarding the presence of small splashes of the oil on their shoes, clothes, or gloves. For trained workers, using properly-ventilated equipment, the hazard consists chiefly of accidents in the machinery, with sudden massive exposure to the oil or its vapour. In my experience, there have been only two cases of marked methaemoglobinaemia, and both recovered with no other treatment than removal of contaminated clothing, rest in bed, and oxygen. In the aniline dye industry, I am informed, cases showing cyanosis of the lips are seen almost every day, and are regarded lightly, except when accompanied by some other intoxication, such as the ingestion of alcohol. In the dye industry, also, exposure to dimethyl aniline is sufficiently intense to produce methaemoglobinaemia similar in every way to that caused by aniline; in my own experience dimethyl aniline has never caused any symptoms, probably because it was not extensively handled.

Halocrine.-In the manufacture of mepacrine (quinacrine dihydrochloride), many of the intermediate products are very active, and may constitute primary irritants or sensitizers. In my experience, the intermediate halocrine,<smiles>COc1ccc2nc3cc(Cl)ccc3c(Cl)c2c1</smiles>

has demonstrated a marked ability to sensitize the skin of susceptible workers. Exposure to the chemical dust in some of these men caused a severe contact dermatitis, necessitating permanent transfer to other work.

\section{Final Products of Synthesis}

Mepacrine and acriflavine.-Among the final products of organic synthesis, mepacrine itself deserves mention as an especially troublesome primary irritant and sensitizer. Rather extensive compressing and grinding, thrice repeated, are necessary to prepare the material in the form of tablets; dust control during these operations is mandatory, but extremely difficult, because of the structure of the machines involved. In a study made at the Abbott Laboratories (Watrous, 1944), exposure to the dust at levels between 0.35 and 4.2 microgrammes per litre was sufficient to cause conjunctivitis in more than half of the workmen, and dermatitis, rhinitis, and stomatitis in about a third. Similar symptoms have recently been described by Barlow and others (1946). Acriflavine, another dye of the acridine series, possesses similar, though weaker, irritant properties; workers bottling tablets of this dye often suffer from a conjunctivitis of considerable severity if they transfer the material to their eyes by injudicious rubbing with dust-stained hands.

Nicotinic acid.-Nicotinic acid and its salts have a peculiar effect upon the skin of certain workers who handle these chemicals in bulk: this consists of a diffuse erythema on the exposed parts of the skin, usually not accompanied by itching, and resembling sunburn in appearance. It is usually transient, disappearing in from twelve to twentyfour hours, though sometimes it assumes a papular character on the second day and lasts several days. The amide of nicotinic acid has never produced this rash in my experience (Watrous, 1939).

Powdered penicillin.-Dry powdered penicillin has proved to be a potent sensitizer and skin irritant in all the American factories where it is handled. It may produce follicular erythema, diffuse papular rashes on the exposed parts of the body, or, in some cases, severe generalized urticaria which persists for days or weeks after exposure ceases. In the latter cases it seems probable that a generalized sensitivity has been created by previous contact with the substance, and that the urticaria results from systemic absorption of the powdered drug by inhalation. In a series of four cases showing industrial dermatitis from exposure to penicillin, Friedlaender and others (1946) found the patients to be sensitive to pure crystalline penicillin rather than to any particular impurity in the commercial product.

Local anaesthetics.-The local anaesthetics, procaine, butyn (butecaine sulphate), and butesin ( $n$-butyl aminobenzoate), form a group of substances more or less prone to cause sensitization and dermatitis upon prolonged contact with the skin. The tendency for procaine to sensitize the skin of dentists and others who frequently spilled solutions on their hands was recognized many years ago, and was one of the factors which made prepared solutions in cartridges so popular with dentists. Women engaged in filling cartridges with sterile solutions of procaine are subject to a characteristic occupational dermatitis caused by excess solution overflowing from the tubes and running down their arms. This usually mild, papular dermatitis is specifically located along the path taken by the drops as they 
run from the wrist to the elbow on the flexor surface of the forearm. It may be prevented by equipping the workers with rubber gloves with turned-up cuffs to catch the liquid; it promptly subsides when contact with the solution ceases. Some operators may be returned to their work after the dermatitis subsides, and appear to suffer no further inconvenience. Others experience a recurrence of the condition in a more severe form, and are thus classified as permanently excluded from work with procaine. Butyn and butesin have produced much less industrial dermatitis in my experience, possibly because they are marketed in forms not requiring such intimate contact with the skin in the process of manufacture.

Neoarsphenamine.-Neoarsphenamine, apart from its arsenic content, may occasionally provoke sensitization of the skin in those who pack it in ampoules. In such cases the minute amount of dust which settles on the skin during the process may cause papular rashes on the exposed skin areas. In one such case the skin was so sensitive to contact with the drug that a patch test made with a few milligrammes of the powder exhibited vesiculation within twelve hours. Such marked reactions, however, are rare; patch tests are usually negative, but many mild dermatoses of this kind subside when contact with the drug ceases and recur when it is resumed. Neoarsphenamine may also act as a primary irritant if it is allowed to accumulate under the nails of those handling it. This is especially prone to occur in women who affix labels to the sealed ampoules. Occasionally an ampoule breaks in the machine, and the spilled powder is wiped up with a damp cloth. The cloth soon becomes stained deep brown as the wet powder oxidizes. If this oxidized material accumulates beneath the nails of the operators, a deep brown stain is produced, and the distal edge of the nail becomes exquisitely tender, with signs of inflammation extending a few millimetres proximally. Hot soaks of 10 per cent. sodium thiosulphate, with temporary change of work, usually bring the condition under control. . To prevent it the wiping rags should be moistened with sodium thiosulphate and should be frequently changed.

2-methyl-1,4-naphthoquinone.-2-methyl-1,4-naphthoquinone, a synthetic form of vitamin $K$, has the property of sensitizing the skin and producing dermatitis. Workers who handle this chemical present a dark-brown staining of the skin, and may later show papular or eczematoid dermatitis. These phenomena were noted in the works at the time when the chemical was first being prepared for clinical use, but were not considered significant, since the medica- tion was to be administered orally. However, Page and Bercovitz (1942), who had been successful in administering 2-methyl-1,4-naphthoquinone to infants by percutaneous inunction, encountered dermatitis in five out of nine adults to whom they gave the material by the same route. Itching, erythema, oedema of the skin, and even ulceration were observed in some of the subjects.

Sulphonechloramides.-At least two sulphonechloramides which are used as antiseptics or disinfectants have rather unusual properties as local irritants and sensitizers. These are chloramine- $T$

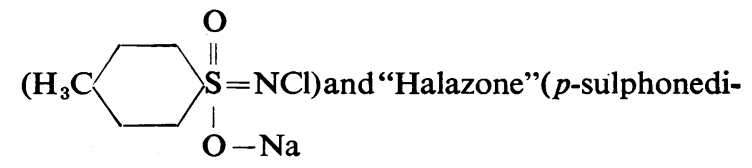

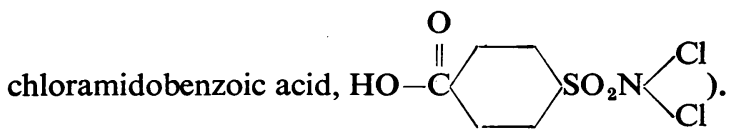

When these chemicals contaminate the working environment in the form of dust, they may produce dermatitis, rhinitis, conjunctivitis, and bronchitis in their capacity as primary irritants. Their most remarkable property, however, is their ability to produce specific atophy in certain individuals. These workers, after rather heavy exposure to the chemicals, gradually become more and more intolerant to them, and begin to exhibit typical asthmatic symptoms instead of the initial picture of simple irritative bronchitis. Finally, as the syndrome is fully developed, they are unable to encounter the minutest quantities of the chemicals in the air without suffering an asthmatic attack.

A series of fourteen such patients, investigated by Feinberg and Watrous (1945) all gave whealing responses to scratch tests performed with chloramine-T solutions in dilutions ranging from 1:10 to 1:10,000. Four of the more sensitive patients had reagins in their blood, which were demonstrated by passive transfer. In other words, these patients gave every reaction to this simple chemical compound which one finds in typical allergies of the atophic variety, formerly believed to be provoked exclusively by proteins of high molecular weight. In view of the serious and permanent disability imposed by asthma, no pharmaceutical workers should ever be subjected to heavy exposure to chemicals of this general type.

Mercury poisoning.-The hazard of mercury poisoning must be considered in the pharmaceutical industry in connexion with three occupations: the manufacture of organic mercurial antiseptics, the moulding of mercury bichloride and mercury oxycyanide tablets, and the use of mercury manometers. In making solutions of organic mercurial 
antiseptics, it is often necessary to allow dilute solutions to age for several weeks in 1,000-gallon tanks to eliminate precipitation when the product is finally filled into bottles. It is not unusual to observe a fine film of tiny droplets of metallic mercury on the glass lining of the manholes of such tanks-ample evidence that the atmosphere above the solution must contain mercury vapour. In my experience, no cases of poisoning have ever resulted from this particular cause, but under unusual conditions there is no doubt that the possibility of mercurial poisoning would be present.

In the moulding of antiseptic tablets of mercury bichloride or mercury oxycyanide, a moist powder or mass is rubbed into a mould with a spatula. The tablets are then ejected by means of a male punch-plate, spread on trays, and dried in an oven. In another method the tablets are punched on a machine, using a dry granulated form of mercury bichloride. In either case the chief danger is systemic absorption of mercury by inhalation of dust; minor symptoms may be caused by the local irritant action of mercury salts on the skin. Tabletmoulders should be carefully selected from a group of older, responsible employees who have had enough experience to develop a wholesome respect for and knowledge of poisons. They should be supplied with efficient dust masks and rubber gloves, and should carry out their moulding under ventilated hoods. The unloading of dried tablets should be done in hoods, and any subsequent counting and bottling must be surrounded with even more stringent precautions. When such precautions are in effect, air levels of mercury are of the order of $0.002 \mathrm{mg}$. per $\mathrm{cu}$. $\mathrm{ft}$. Under such conditions no cases of mercurialism have come to my notice.

In the laboratory, or in certain manufacturing operations, mercury offers peculiar advantages as a liquid of high specific gravity. It finds extensive use in manometers, in apparatus for gas analysis, and in simple devices for maintaining inert gases at a constant positive pressure. The latter instance has considerable manufacturing importance in filling ampoules which must be protected by a layer of inert gas above the liquid or solid. To obtain a constant flow of inert gas from high-pressure cylinders, some type of pressure-release must be provided; a tube immersed in a few inches of mercury is effective for this purpose. The constant bubbling of the gas through the liquid must carry some mercury vapour into the work-room, and there is, of course, the inevitable spillage which occurs when such devices are adjusted or moved frequently. Here, as in chemical laboratories, one cannot assume the concentration of mercury vapour to be insignificant; actual measurement of the air content should be made periodically to be sure this is so.

Stilboestrol.-The chemical synthesis of diethylstilboestrol is hazardous in its final stages, in which the drug is purified by crystallization from various fat solvents such as ether and benzene. As Fitzsimons (1944) has pointed out, spillage of these concentrated solutions on the skin may lead to direct absorption of toxic amounts of stilboestrol, with the production of gynaecomastia in male workers. Spilling small amounts of the solution on workbenches leaves a deposit of fine powder which may contaminate the air as dust.

To Fitzsimons's twenty cases I can add two more which I observed in young men who purified diethylstilboestrol, and several others of similar causation and course have been reported to me (Passarelli, personal communication). In these cases the patients complained of moderate to severe loss of libido. In the two men I observed, a moderate normocytic anaemia developed concomitantly with the hypertrophy of the breasts; in one case the red count and haemoglobin fell from 4.4 to 3.7 million and from 15.6 to $12.6 \mathrm{~g}$. per $100 \mathrm{c.cm}$., respectively. In the other case the fall was from 5.0 to 3.9 million and from 16.0 to $14.4 \mathrm{~g}$. This caused alarm in view of the depressant effect of diethylstilboestrol when large doses were given to animals (Von Haam and others, 1941; Castrodale and others, 1941). However, when the patients were removed from contact with the drug, their blood quickly returned to a normal state and the other symptoms gradually subsided.

The physical hazard in exposures of this kind, in factories where good industrial medicine is practised, is probably far less than the psychological hazard. The fact that an industrial poison may have a feminizing effect is something new under the sun, and its impact upon suggestible young men with neurotic leanings can be disastrous. In industries where poor relations obtain between labour and management, or where industrial medical service is rudimentary, exposure to this chemical may be expected to be a rich source of litigation. I know of one instance in which the manufacture of diethylstilboestrol was abandoned for this very reason. It is worthy of note that the mixing and handling of diethylstilboestrol in the diluted forms in which it is dispensed for clinical use have never, in my experience, given rise to any symptoms; the sole danger seems to arise in the handling of the pure chemical.

Morphine and codeine.-Tablets of morphine and codeine intended for hypodermic injection must still be hand-moulded in the pharmaceutical industry, since machine-made tablets would not dissolve quickly enough to be practical. The handmoulding process involves much handling of the raw materials, and under certain conditions enough dust may be created to permit effective doses of these drugs to be absorbed. Cases of serious poisoning can scarcely occur, because of the relatively large amount of material which would have to 
be absorbed by inhalation. However, it is not unusual for tablet workers to complain of drowsiness and lethargy if exposed to unusual amounts of dust. The suspicion that effective doses are absorbed in this way from the respiratory tract is strengthened by the observation that, when mixtures of morphine and atropine are being handled, pupillary dilatation and dry mouth may be observed in addition to the other complaints. No case of addiction, however, has ever come to my notice as a result of such exposure.

Veterinary preparations.-Many pharmaceutical manufacturers make certain products intended for veterinary use, and the physician whose knowledge is confined to the armamentarium of human medicine would little suspect what poisons are still in good repute in the art of healing animals. A tonic powder for horses may contain up to 2.5 per cent. of white arsenic; vermifuge capsules for animals may contain pure tetrachlorethane. Thus it may come to pass that packers are inadvertently exposed to dangerous amounts of arsenic, or soft-capsule makers to toxic vapours, in the innocent belief that material used as 'medicine' can hardly be dangerous! Such errors will be eliminated in the well-organized pharmaceutical works by seeing that the formula department affix special precautionary labels to all formulae containing poisons, and by including in instructions for compounding the formula details of all necessary precautions for handling the ingredients.

\section{Miscellaneous Hazards}

In addition to the specific hazards peculiar to the pharmaceutical industry, workers in this occupation are subjected to a number of other noxious agents common to other industries.

\section{Radiant Energy}

Radiant energy may endanger pharmaceutical workers in several different ways.

Ultra-violet light. - Exposure to ultra-violet light occurs in the arc-welding incident to the construction and repair of manufacturing equipment as well as in rooms devoted to the manufacture of synthetic vitamin D from ergosterol. In addition, so-called germicidal lamps have found certain applications in the packaging of products subject to spoilage from air contamination. The prime factor in the injuries inflicted by these modern devices is ignorance on the part of well-meaning persons who become enthusiastic about ' applying the benefits of modern science' to their tasks without understanding the fundamental principles involved. All radiant energy of wavelength too short to be perceived by the senses produces injury not immediately perceived by the senses, but this is a concept which modern man has not yet appreciated. However, he must learn to appreciate it if he is to survive much longer on this planet. In the near future pharmaceutical industries will probably be occupied with furnishing radio-active isotopes of chemical elements which will localize in specific tissues, such as the thyroid gland and the osseous system, and the safe handling of these substances can be guided only by rational processes, not by any pre-existing or familiar pattern of experience.

Visible light.-In the visual spectrum, an occasional source of complaint by industrial workers is the introduction of fluorescent lighting in workrooms formerly illuminated by incandescent lamps. Complaints of eye-strain, headache, and many other subjective symptoms will arise in suggestible individuals when any change is made in their environment, and such complaints may be disregarded when attributed to fluorescent lighting. The only justifiable complaint with respect to this type of illumination comes from those who work with rapidlymoving objects under lighting which does not include the proper ballast and diphasic circuits to eliminate the stroboscopic effect of fluorescent lamps.

In the pharmaceutical industry, ampoule-sealers are, by virtue of their occupation, specifically exposed to the effect of visible light. Their occupation involves daily gazing into gas flames used for raising glass to fusion temperature, as well as fine discrimination as to the proper temperature at which to draw off the fused neck of the ampoule to make a proper seal. These workers are prone to accept the belief that such work is hard on their eyes, and tend to attribute any. occular defects which may arise in their lives to their peculiar occupation. The majority of sealers elect to wear tinted glasses to reduce the glare from the flames, but the tint cannot be too deep, because it would then diminish visual acuity necessary for handling the ampoules under ordinary illumination.

Glass containing praseodymium, and sold under the trade name didymium glass by the Corning Glass Works, Corning, New York, U.S.A., makes a very effective filter for this particular work. The main component of the glare coming from hot glass in a gas flame is due to the practically monochromatic yellow radiation of the sodium D lines. Didymium glass selectively absorbs this light, but passes enough of the other radiations of the visual spectrum to allow good vision in rather dim surroundings. The elimination of glare from the flame enables the worker to see the molten glass more clearly, and thus should reduce eye-strain.

To test the truth of the workers' frequent assertions that sealing was bad for the eyes, two surveys were made in which several visual functions of the entire sealing group were tested. An interval of one year separated the 
surveys. The instrument used was the Bausch and Lomb ortho-rater, by means of which visual acuity may be measured for far and near objects, phorias may be detected, depth perception measured, and colourblindness revealed. The tests were made on each eye separately, both with and without the corrective glasses, if any were worn. Twenty-seven of the employees tested in the'first survey were available for the second. Of these, twenty-six showed either improved performance or no change in the second test, whereas one showed poorer performance. The great preponderance of those showing improved vision was attributed to the fact that many had become aware of uncorrected visual defects or poorly-fitting glasses in the first survey, and had taken remedial steps.

Infra-red light.-The use of banks of incandescent lamps radiating a high percentage of infra-red energy was applied in the automobile industry to hasten the drying of lacquer on rapidly-moving production lines, and has found at least one application in the pharmaceutical industry for the same purpose. Those who design such devices are rarely aware of the fact that intense infra-red radiation may produce cataract; therefore the industrial physician must inspect such installations and assure himself that no worker receives harmful amounts of this radiation.

\section{Bacteria and Viruses}

A small group of laboratory workers in any large pharmaceutical industry will necessarily undergo an occupational exposure to virulent bacteria and viruses. Even though such employees have had extensive professional training which is supposed to tender them extremely careful, accidents may occur; this is especially true where virulent cultures are handled in large quantities, as in the manufacture of vaccines. Several fatal infections with the virus of equine encephalomyelitis occurred in the United States in connexion with large-scale manufacture of vaccine (Helwig, 1940). As a result, experiments were carried out to determine a suitable immunization procedure to be applied to laboratory workers.

In 1939 a small group of laboratory workers at the Abbott Laboratories were given four injections of Abbott equine encephalomyelitis veterinary vaccine, mixed Eastern and Western types, diluted 1:1 with ' normal salt solution. Each dose consisted of $1 \mathrm{c.cm}$. injected into the gluteal muscle. The doses were repeated at two- to three-day intervals. Six weeks after the vaccination, blood serum from the men in the group was tested for neutralizing power against the living virus. Only a slight increase in titre was observed. Accordingly, each subject received $0.5 \mathrm{c.cm}$. of undiluted vacccine. In 1940, Beard and others described a method which successfully immunized 100 exposed persons; $2 \mathrm{c.cm}$. of full-strength vaccine were injected into the gluteal muscle twice. At present the routine method of immunization at Lederle Laboratories, where this vaccine is still manufactured is to inject $0.5 \mathrm{c.cm}$. of Western type vaccine into one deltoid muscle, and $0.5 \mathrm{c.cm}$. of Eastern type into the other. The same doses are repeated in a week. Thereafter, one 'booster' dose of the same size is given annually (Hardy, personal communication).

\section{Reagents, etc.}

Several toxic hazards arise in large bacteriological or pharmaceutical laboratories where strong reagents, illuminating gas, and large amounts of glassware are used. A popular solution for cleaning glassware is a mixture of sulphuric acid and potassium dichromate. A chemist cleaning his own glassware rarely has enough contact, with this solution to produce disease, but in large laboratories the division of labour tends to create exposures which may produce severe dermatosis. The hands are most affected, and tend to become hyperkeratotic and fissured. Extreme cases of this dermatosis may be seen in old photolithographers, who handle chrome etch with their bare hands.

One case of erosion of the nasal mucous membranes arose from the use of this solution as the result of a single accident. A well-meaning, but chemically naïve worker thought she might prevent contact of the solution and her hands by using a tray for the glassware, which could be immersed in the concentrated acid. Unfortunately the model tray which was used to try out the idea was made of iron galvanized with zinc. The violent evolution of hydrogen created a mist of acid chromate solution, which was inhaled with painful, though educational, results. Trays were later made of stainless steel.

Another laboratory hazard attends the use of glass wool, or of cloth made of spun glass fibre. In the preparation of packages for the administration of blood plasma during the war, rolled tape made of glass cloth was used in the filter assembly. Workers who cut and rolled this tape developed irritation of the skin on the flexor surfaces of the forearms, where their arms rested on the table top. This was eliminated by providing long cuffs of cellulose acetate.

Blood-plasma preparation required a few workers to spend almost half their time inside large refrigerators. The blood was centrifuged in cold rooms at about $40^{\circ} \mathrm{F}$., and frozen plasma was stored in rooms close to $0^{\circ} \mathrm{F}$. From these rooms, the workers would emerge into a working area at 70 to $80^{\circ} \mathrm{F}$., and it was this frequent transition which. caused all their complaints. Many assertions were made that this work was bad for the health, and that it caused frequent colds and coughs. However, the hazard was more mental than physical, and no increase in respiratory infections could be demonstrated objectively. 
Chemical laboratory research workers are génerally considered too well educated to do anything so foolish as poison themselves with the reagents they use. In my experience this assumption is unwarranted. Where such workers are exposed to benzene vapour, they require just as careful watching as any chemical labourer. One pharmaceutical manufacturer found leukopenia in five out of twenty research chemists. Most of these men had Ph.D. degrees, but they had received no instruction or special warning during their university studies and were unaware of any particular hazard in handling benzene. In reporting this, the Industrial Hygiene Division of the United States Public Health Service (Bloomfield, 1945) calls for adequate instruction in American universities concerning laboratory precautions for chemical research workers.

Miscellaneous hazards are encountered by the mechanics who install and repair the great variety of equipment needed in pharmaceutical works. Many large vessels used for chemical reactions must be lined with sheet lead, and the fabricating and repair of such linings is a skilled trade. Lead burners, because of this special skill, are likely to be steadily occupied handling lead only; they scrape, brush, file, and weld the sheet. The technique employed for welding lead sheet in the Abbott works requires the use of an oxy-hydrogen torch. This burns with a quiet, moderately hot flame, and has no tendency whatever to produce lead oxide fume; indeed, the flame seems to possess reducing properties which prevent any oxidation of lead. The scraping, brushing, and filing of the sheet produce mainly large particles which have no tendency to remain suspended in the air; however, the handling of old, corroded lead sheet would probably involve more dust. Six urinary lead determinations have been made on one lead burner over a period of nineteen months. These varied between 0.015 and $0.037 \mathrm{mg}$. per day. No symptoms of plumbism have appeared. The use of oxy-acetylene torches in fusing lead gives rise to abundant fume, and would be more hazardous. Other fumes encountered by maintenance workers include zinc and cadmium oxide, which are generated when iron pipes plated with these materials are heated. The very toxic effect of cadmium fumes, not generally known to the average shop worker, makes it imperative to supervise all use of cadmium-plated pipe very closely.

\section{Conclusion}

It should be apparent from the foregoing that any pharmaceutical industry, large or small, is particularly in need of expert counsel in matters affecting the health of workmen. A thorough knowledge of the literature of known poisons is essential for the industrial specialist in this field, but he must also have an alert readiness to detect and investigate possible new poisons. In doing so, the process of untangling the threads of a new syndrome from the inevitable surrounding mass of subjective symptoms, neurotic complaints, incidental diseases, and other irrelevancies requires the nicest judgment and scientific balance, together with a most intimate knowledge of all the manufacturing processes. Far as one may be from reaching this goal, the struggle towards it is full of interest and satisfaction.

\section{REFERENCES}

Astwood, E. B., Bissell, A., and Hughes, A. M. (1945). Endocrino$\log y, 37,456$.

Barlow, O. W., Gorhan, L. W., and Bedell, A. J. (1946). Occup. Med., 1, 482.

Beard, J. W., Beard, D., and Finkelstein, H. (1940). J. Immunol., 38, 117.

Bloomfield, J. J. (Ed.) (1945). Industr. Hyg. News Letter (U.S. Publ. Hith. Service), vol. 5, No. 10, p. 2

Brown H. (1941) U.S. Bureau of Mines, I. C. No. 7148: Dust Explosion Hazards in Plants Producing or Handling Aluminium, Magnesion or Zinc Powder.

Castrodale, D., Bierbaum, O., Helwig, E. B., and Macbryde, C. M. (1941). Endocrinology, 29, 363.

Chen, K. K., Rose, C. L., and Clowes, G. H. A. (1944). J. Indiana State med. Assoc., 37, 344.

Deichmann, W B., Kettering Laboratories, Cincinnati. Personal communication.

Feinberg, S. M., and Watrous, R. M. (1945). J. Allergy, 16, 209.

Fitzsimons, M. P. (1944). Brit. J. industr. Med., 1, 235.

Flury, F., and Zernik, F. (1931). 'Schädliche Gase.' Julius Springer, Berlin.

Friedlaender, A. S., Watrous, R. M., and Feinberg, S. M. (1946)。 Arch. Derm. Syph., Chicago. 54, 517.

Greenburg, L., Mayers, M. R., Goldwater, L., and Smith, A. R. (1939), J. industr. Hyg., 21, 395.

J. industr. Hyg., 21, 395.
Haam, E. von, Hammel, M. A., Rardin, T. E., and Schoene, R. H. (1941). Endocrinology, 28, 263.

Hager, G. P., Eli Lilly and Co. Personal communication.

Hardy, S. M., Lederle Laboratories. Personal communication.

Heffter, A. (1923). 'Handbuch der experimentellen Pharmakologie.' Julius Springer, Berlin. Vol. 1, p. 798.

Helwig, F. C. (1940). J. Amer. med. Ass., 115, 291.

Letter from Paris (1946.) Ibid., 132, 236.

Page, R. C., and Bercovitz, Z. (1942). Amer. J. med. Sci., 203, 566.

Passarelli, E. W. (Chicago). Personal communication.

Pechmann, $\mathbf{H}$. von (1895). Chem. Berichte, 28, 855.

Perrault, M., Bovet, D., Droguet, P., and Jeantet. (1944-5). Arch. Malad. profess., 6, 245.

Peshkin, M. M. (1920). J. Amer. med. Ass., 75, 1,133.

Richards, R. K. Department of Pharmacology, Abbott Laboratories. Personal communication.

Salter, H. H. (1860). 'On Asthma.' J. and A. Churchill, London.

Salter, H. H. (1860). 'On Asthma.' J. and A. Churchi
Watrous, R. M. (1939). J. Amer. med. Ass., 112, 2132.

Watrous, R. M. (1939). J. Amer. med.

(1942). Industr. Med.,

(1943a). Ibid., 12, 721 . Ibid., 12, 832.

(1943b). Ibid., 13, 887.

, and McCaughey, M. B. (1945). Ibid., 14, 639. 